\title{
Identification of the biological function of miR-9 in spinal cord ischemia-reperfusion injury in rats
}

\author{
Fengshou Chen ${ }^{1}$, Jie Han ${ }^{1}$, Xiaoqian Li ${ }^{1}$, Zaili Zhang ${ }^{1}$, Dan Wang ${ }^{\text {Corresp. } 1}$ \\ ${ }^{1}$ Department of Anesthesiology, the First Hospital of China Medical University, Shenyang, Liaoning, China \\ Corresponding Author: Dan Wang \\ Email address: wonder12251@hotmail.com
}

Spinal cord ischemia-reperfusion injury (SCII) is still a serious problem, and the mechanism is not fully elaborated. In the rat SCII model, qRT-PCR was applied to explore the altered expression of miR-9 (miR-9a-5p) after SCll. The biological function of miR-9 and its potential target genes based on bioinformatics analysis and experiment validation in SCII were explored next. Before the surgical procedure of SCII, miR-9 mimic and inhibitor were intrathecally infused. miR-9 mimic improved neurological function. In addition, miR-9 mimic reduced blood-spinal cord barrier (BSCB) disruption, inhibited apoptosis and decreased the expression of IL- 6 and IL- $\beta$ after SCII. Gene Ontology (GO) analysis demonstrated that the potential target genes of miR-9 were notably enriched in several biological processes, such as "central nervous system development", "regulation of growth" and "response to cytokine". The Kyoto Encyclopedia of Genes and Genomes (KEGG) analysis revealed that the potential target genes of miR-9 were significantly enriched in several signaling pathways, including "Notch signaling pathway", "MAPK signaling pathway", "Focal adhesion" and "Prolactin signaling pathway". We further found that the protein expression of MAP2K3 and Notch2 were upregulated after SCll while miR-9 mimic reduced the increase of MAP2K3 and Notch2 protein. miR-9 mimic or MAP2K3 inhibitor reduced the release of IL- 6 and IL-1ß. miR-9 mimic or si-Notch2 reduced the increase of cleaved-caspase-3. Moreover, MAP2K3 inhibitor and si-Notch2 reversed the effects of miR-9 inhibitor. In conclusion, overexpression of miR-9 improves neurological outcomes after SCII and might inhibit BSCB disruption, neuroinflammation, and apoptosis through MAP2K3-, or Notch2-mediated signaling pathway in SCII. 
1 Identification of the biological function of miR-9 in spinal cord ischemia-reperfusion injury

2 in rats

3 Fengshou Chen ${ }^{1}$, Jie Han ${ }^{1}$, Xiaoqian Li $^{1}$, Zaili Zhang ${ }^{1}$, Dan Wang ${ }^{1}$

$4{ }^{1}$ Department of Anesthesiology, the First Hospital of China Medical University, Shenyang,

$5 \quad$ Liaoning province, China

6 Corresponding author:

7 Dan Wang

8 Address: Department of Anesthesiology, the First Affiliated Hospital of China

9 Medical University, Shenyang, Liaoning province, China.

10 Email: wonder12251@hotmail.com

\section{Abstract}

12 Spinal cord ischemia-reperfusion injury (SCII) is still a serious problem, and the mechanism is not fully elaborated. In the rat SCII model, qRT-PCR was applied to explore the altered expression of miR-9 (miR-9a-5p) after SCII. The biological function of miR-9 and its potential target genes based on bioinformatics analysis and experiment validation in SCII were explored next. Before the surgical procedure of SCII, miR-9 mimic and inhibitor were intrathecally infused. miR-9 mimic improved neurological function. In addition, miR-9 mimic reduced bloodspinal cord barrier (BSCB) disruption, inhibited apoptosis and decreased the expression of IL-6 and IL- $\beta$ after SCII. Gene Ontology (GO) analysis demonstrated that the potential target genes of miR-9 were notably enriched in several biological processes, such as "central nervous system development", "regulation of growth" and "response to cytokine". The Kyoto Encyclopedia of Genes and Genomes (KEGG) analysis revealed that the potential target genes of miR-9 were significantly enriched in several signaling pathways, including "Notch signaling pathway", "MAPK signaling pathway", "Focal adhesion" and "Prolactin signaling pathway". We further found that the protein expression of MAP2K3 and Notch2 were upregulated after SCII while miR-9 mimic reduced the increase of MAP2K3 and Notch2 protein. miR-9 mimic or MAP2K3 inhibitor reduced the release of IL-6 and IL-1 $\beta$. miR-9 mimic or si-Notch2 reduced the increase 
of cleaved-caspase-3. Moreover, MAP2K3 inhibitor and si-Notch2 reversed the effects of miR-9 inhibitor. In conclusion, overexpression of miR-9 improves neurological outcomes after SCII and might inhibit BSCB disruption, neuroinflammation, and apoptosis through MAP2K3-, or Notch2-mediated signaling pathway in SCII.

Key words:Spinal cord ischemia-reperfusion injury;miRNA9 ;MAP2K3; Notch2; apoptosis; neuroinflammation

\section{Introduction}

Spinal cord ischemia reperfusion injury (SCII) is a complication occurring under thoracoabdominal aortic or spinal cord surgery, which brings the risk of paralysis and paraplegia(Li et al. 2015a; Xu \& Li 2020). Although various measures were developed and applied to reduce the risks and improve the outcomes of SCII, satisfactory therapeutic effects still not be achieved due to multifactorial pathogenic factors(Ege et al. 2004; Fang et al. 2015; Li et al. 2014a; Li et al. 2014b; Zhou et al. 2013b).

MicroRNAs (miRNAs, miRs) are 21- to 23- nucleotide noncoding RNA that are capable of specific binding mRNA and regulate post-transcriptional expression(Chen et al. 2020a; He et al. 2015; Li et al. 2016b). It has been discovered that miRNAs might be related to central nervous system (CNS) injury including SCII(Bao et al. 2018; Chi et al. 2014; Huang et al. 2015; Li et al. 2015; Yao et al. 2018). miR-27a mimics reduced blood-spinal cord barrier (BSCB) damage induced by neuroinflammation following SCII via inhibiting the NF- $\mathrm{B} / \mathrm{IL}-1 \beta$ pathway and negatively regulating the TICAM-2 of the TLR4 signaling pathway(Li et al. 2015). In rat models of SCII, miR-125b mimic was found to protect against SCII via reducing aberrant p53 network activation-induced apoptosis and neuroinflammation through the downregulation of TP53INP1(Li et al. 2018c). Several studies have demonstrated altered miRNA expression profiles in spinal cord tissues of SCII models, which implicated the important roles of miRNAs 
55

56

57

in the pathophysiological mechanism of SCII(Hu et al. 2013; Li et al. 2016a; Liu et al. 2020). miR-9 (miR-9a-5p) is a highly conserved mature miRNA across species and serves as a tumour regulator in several cancer types, including colorectal cancer, gastric cancer and breast cancer(Gao et al. 2017; Wang et al. 2015; Yang et al. 2019; Zhu et al. 2015). Evidence also showed that miR-9 is highly expressed in the brain, that is involved in ischemic stroke, transient cerebral ischemia, amyotrophic lateral sclerosis (ALS) (Altintas et al. 2016; Cao et al. 2020; Hawley et al. 2019). Knockdown of TUG1 promoted cell survival and decreased cell apoptosis through increasing the expression of miR-9 and inhibiting the expression of $\mathrm{Bcl} 2$ following brain ischemia(Chen et al. 2017). A recent study has shown that ferulic acid treatment protected against neuronal death in the rat hippocampus following hypoxic-ischemic damage through the inhibition of miR-9 induction(Yao et al. 2020). However, whether miR-9 exerts an effect on SCII is unknown.

The aim of the present study was to explore the biological function of miR-9 in SCII and clarify the mechanism via combining bioinformatics analysis and experiment validation.

\section{Materials and Methods}

\section{Experimental animals}

Sprague-Dawley rats (male, 200-250g) were obtained in the present study. All rats were maintained for at least 1 week before the surgical procedures, with freely available rodent chow and water at $22-24^{\circ} \mathrm{C}$ and $50-60 \%$ relative humidity, under a $12 \mathrm{~h} / 12 \mathrm{~h}$ light-dark cycle. The present study had approval from the Ethics Committee of China Medical University, Shenyang (CMU 2020391), and were carried out in conformity with the National Institutes of Health Guide for the Use and Care of Laboratory Animals (NIH Publications No.80-23, revised 1996).

\section{Rat model}

To create SCII rat models, a cross-clamped aortic arch was used as previously reported(Chen et al. 2020a; Chen et al. 2020b). Pentobarbital sodium (50 mg/kg) was intraperitoneal injected for anesthetizing rats. SCII was induced by the aorta occlusion for $14 \mathrm{~min}$. The same surgical 
81 procedures without occlusion were performed on sham-operated rats.

82

\section{Euthanization}

Rats were euthanized by sevoflurane overdose and ensured that it was effective by pinching the tail with tweezers. Any movement in the rat showed that pain could still be felt so enough time was allowed for the anesthesia to fully work before sacrificing the mice. Rats that survived the study or were excluded were bred for other experiments.

\section{Quantification of miRNA expression}

Total RNA was extracted from segments L4-L6 of the spinal cord with using Trizol reagent (Takara, Otsu, Japan). RNA was reverse-transcribed into cDNA using the Prime Script ${ }^{\circledR}$ miRNA cDNA Synthesis Kit (Takara, Tokyo, Japan) (Li et al. 2016b; Wang et al. 2015). The levels of miR-9 were detected using SYBR Premix qRT-PCR on a PCR System (Corbett Research, Australia) (Chen et al. 2020a). The primers used for miR-9 in the present study were as follows: forward: 5'- CGCGCTCTTTGGTTATCTAGCTGTATG -3'(Yao et al. 2018). Relative miR-9 expression was normalized to U6 expression levels using the $2^{-\Delta \Delta \mathrm{Ct}}$ method.

\section{Intrathecal administration}

Thoracic laminectomy was performed for intrathecal pretreatment(Li et al. 2015). Pretreatment with a synthesized miR-9 mimic (5'- UCUUUGGUUAUCUAGCUGUAUGA -3'), inhibitor(5'-TCATACAGCTAGATAACCAAAGA-3') and negative control (NC) was previously described(Li et al. 2015). Rats were intrathecally injected with $10 \mu$ of the oligonucleotides $(500 \mathrm{pmol} / 10 \mu \mathrm{l})$ and EntransterTM-in vivo transfection regent (Engreen, Beijing, China) (Wang et al. 2020). Intrathecal injection was applied in vivo prior to ischemia induction once a day for three consecutive days according to the results of our preliminary experiment(Li et al. 2016b; $\mathrm{Li}$ et al. 2015). In order to inhibit the expression of MAP2K3, SB203580 (5 $\mu$ l, dissolved in $0.1 \mathrm{nmol} / \mu \mathrm{l}$ solution using $1 \%$ DMSO) once daily for 2 consecutive days (Yao et al. 2018; Zhou et al. 2016) at the same time. Notch2 siRNA or NC RNA were provided by Jima Inc. (Shanghai China). For suppressing the expression of Notch2, 
107 two days before ischemia, intrathecal infusion of $5 \mu \mathrm{g}$ of siRNA(5'-

108

109

110

111

112

113

114

115

116

117

118

119

120

121

122

123

124

125

126

127

128

129

130

131

132

133

CCTCCCATCGTGACTTTCCAGCTTA-3') or control RNA at a concentration of $1 \mu \mathrm{g} / \mu$ l once a day was carried out, as directed by the manufacturer(Chen et al. 2020b; Meng et al. 2015; Wang et al. 2020).

\section{Experimental protocol}

Protocol I

To measure expression of miR-9 at various time points $(24,48$ and $72 \mathrm{~h})$ after reperfusion, rats were euthanized at each time point after SCII $(n=3)$. Rats were assigned to four groups $(n=8)$ : (1) Sham group; (2) SCII group; (3) SCII+NC group; (4) miR-9 mimic group. miR-9 NC or miR-9 mimics was injected intrathecally prior to SCII induction once a day for three consecutive days in the SCII+NC group or miR-9 mimic group, respectively(Li et al. 2015; Wang et al. 2020).

Protocol II

Rats were assigned to eight groups( $\mathrm{n}=8)$ : (1) Sham group; (2) SCII group; (3) SCII+NC group; (4) miR-9 mimic group; (5) miR-9 inhibitor group; (6) SB203580 + miR-9 inhibitor group; (7) SB203580 group; (8) DMSO group. In the SB203580 + miR-9 inhibitor group, except for miR-9 inhibitor pretreatment, SB203580 (5 $\mu$ l, dissolved in $0.1 \mathrm{nmol} / \mu \mathrm{l}$ solution using $1 \% \mathrm{DMSO}$ ) once daily was injected intrathecally once a day for two consecutive days before SCII(Yao et al. 2018; Zhou et al. 2016). SB203580 or DMSO was injected intrathecally prior to SCII induction once a day for two consecutive days in the SB203580 group or DMSO group, respectively.

\section{Protocol III}

Rats were assigned to eight groups $(\mathrm{n}=8):(1)-(5)$ The first five groups are the same as those in Protocol II; (6) si-Notch2 + miR-9 inhibitor group; (7) si-Notch2 group; (8) siRNA NC group. In the si-Notch2 + miR-9 inhibitor group, except for miR-9 inhibitor pretreatment, si-Notch2 $5 \mu \mathrm{g}$ at a concentration of $1 \mu \mathrm{g} / \mu \mathrm{l}$ was injected intrathecally once a day for two consecutive days before SCII except for miR-9 inhibitor pretreatment(Chen et al. 2020b; Meng et al. 2015; Wang et al. 2020). si-Notch2 or siRNA NC was injected intrathecally prior to SCII induction once a 
134 day for two consecutive days in the si-Notch2 group or siRNA NC group, respectively.

135 Neurological evaluation

136 Two observers evaluated the movement function of the rat lower limb based on the BBB scoring 137 after SCII as described previously(Bao et al. 2018; Basso et al. 1995).

\section{Bioinformatics analysis for potential target genes of miR-9}

At first, targets of miR-9 were predicted by means of databases miRDB and Targetscan. Target genes were identified for miR-9 in both databases. Then the raw data of GEO Series (GSE)138966 [species: Rattus norvegicus; Platforms: GPL22396Illumina HiSeq 4000 (Rattus norvegicus)] was obtained from Gene Expression Omnibus database (http://www.ncbi.nlm.nih.gov/geo/). 3 sham-operated samples and 3 SCII samples at 48 h postSCII were included in GSE138966(Ding et al. 2020). The differentially expressed genes (DEGs) between sham-operated samples and SCII samples were obtained. DEGs are those genes with an $|\log 2 \mathrm{FC}| \geq 1$ and $p<0.05$. For the purpose of analyzing the diversification of DEGs expression, the heatmaps of DEGs were drawn by heatmap function. We chose the intersection of the upregulated DEGs and the above predicted genes as the potential miR-9 target genes and conducted bioinformatics analysis.

DAVID 6.8 (https://david.ncifcrf.gov/) is a database with annotation, visualization, and integrated discovery functions(Huang da et al. 2009). The annotation table is its main analysis tool, which contains functional annotation charts, functional annotation clustering, and functional annotation table subtools (Wang et al. 2021). Gene Ontology (GO) and Kyoto Encyclopedia of Genes and Genomes (KEGG) annotation of DEGs was carried out through the annotation tool. The biological functional coherence and biological attributes of the putative target genes were determined based on GO analysis. KEGG is a collection of databases dealing with genomes, diseases, biological pathways, drugs and chemical materials(Altintas et al. 2016). The biological processes and pathways of potential miR-9 target genes were analyzed using the DAVID online database. $p<0.05$ was selected as the cut-off criterion with statistic difference. According to the relations of genes and statistically significant pathways as well as the relations among genes, pathways and miR-9, we also built a miRNA-pathway-gene network 
162

163

164

165

166

167

168

169

170

171

172

173

174

175

176

177

178

179

180

181

182

183

184

185

\section{Evans blue (EB) extravasation}

EB cannot normally pass through $\mathrm{BSCB}$ and thus its presence in spinal cord tissue indicates BSCB disruption (Goldim et al. 2019). At the time point of $48 \mathrm{~h}$ after SCII, the intravenous injection of EB (45 mg/kg) was performed. The rats were euthanized after $1 \mathrm{~h}$ circulation. Segments L4-L6 were homogenized with trichloroacetic acid, and the tissues were centrifuged (Li et al. 2015). The absorption of the supernatant was measured at $632 \mathrm{~nm}$ with a microplate reader (BioTek, Winooski, USA) (Fang et al. 2015). EB staining was visualized using a fluorescent microscopy (Leica, German) with a green filter(Chen et al. 2020a).

\section{Water content of spinal cord}

Water content (\%) of segments L4-L6 was obtained using the following formula: (wet weight - dry weight $) /$ wet weight $\times 100 \%(\mathrm{Li}$ et al. 2018b). Spinal cord tissues were rapidly removed to measure wet weight. Then at $105^{\circ} \mathrm{C}$ for $48 \mathrm{~h}$ the tissues were dried and measure dry weight.

\section{TUNEL (terminal deoxynucleotidyl transferase-mediated dUTP-biotin nick end labeling)} Assay

10-mm-thick sections were subjected to fluorometric TUNEL assay using the commercial kit (Beyotime) to detect the apoptotic DNA strand breaks(Bao et al. 2018). The sections were fixed with $4 \%$ neutral-buffered formaldehyde for $20 \mathrm{~min}$ and incubated with proteinase $\mathrm{K}$ for $20 \mathrm{~min}$, followed by the labeling reaction for $2 \mathrm{~h}$. Then, the nuclei were stained with DAPI and images captured by fluorescence microscopy.

\section{Western Blotting}

The protein expression levels of MAP2K3, Notch2 in segments L4-L6 of spinal cord tissues were detected with Western Blotting. Spinal cord tissues were collected at $48 \mathrm{~h}$ after SCII. Total proteins were extracted by using protein lysis buffer. Rabbit monoclonal anti-MAP2K3 (1:5000, 
186

187

188

189

190

191

192

193

194

195

196

197

198

199

200

201

202

203

204

205

206

207

208

209

abcam,), rabbit monoclonal anti-Notch2 (1:500, Santcruz), rabbit monoclonal anti-cleaved caspase-3 (1:500, abcam) and HRP-conjugated secondary antibodies (Beyotime, China) were used.

\section{Enzyme-linked immunosorbent assays (ELISAs)}

The rats were euthanized at $48 \mathrm{~h}$ after SCII. ELISA kits purchased from Signalway Antibody Company (College Park, USA) were used to test the levels of IL-6 and IL- $\beta$ according to the instructions of the manufacturer.

\section{Statistical analysis}

Data were expressed as mean \pm standard deviation and analyzed by SPSS 15.0 (IBM, USA). All variables were calculated with one-way ANOVA followed by the Newman-Keuls post hoc test. $p<0.05$ was defined significant.

\section{Results}

\section{Expression of miR-9}

At first, we verified the expression of miR-9 at 24, 48 and $72 \mathrm{~h}$ after SCII by qRT-PCR. The results showed that miR-9 levels significantly decreased at 24 and $48 \mathrm{~h}(p<0.01)$. At $48 \mathrm{~h}$ after SCII, miR-9 expression demonstrated the lowest level $(p<0.01)$, as presented in Figure 1A. These results implicated that aberrant expression of miR-9 might be related with SCII.

\section{miR-9 mimic improved neurological function following SCII}

At $48 \mathrm{~h}$ after SCII, we assessed the levels of miR-9 by qRT-PCR after intrathecal injection of miR-9 mimic for 3 days. miR-9 mimic significantly increased the expression of miR-9 $(p<0.05)$ (Figure 1B). In addition, SCII induced severe neurological deficits of lower extremities $(p<$ $0.01)$ and miR-9 mimic improved neurological function after SCII $(p<0.01)$ according to BBB scores (Figure 1C).

\section{miR-9 mimic attenuated BSCB leakage following SCII}


210 Under a fluorescent microscope, EB extravasation exhibits red color(Fang et al. 2015; Li et

211 al. 2014). EB extravasation at $48 \mathrm{~h}$ after SCII was more ( $p<0.01$ versus the sham rats). In

212 contrast, EB extravasation was reduced with miR-9 mimic pretreatment $(p<0.01)$, as depicted in

213 Figure 2A. EB fluorescence density and content were calculated, as depicted in Figure 2B and

214 2C. Spinal cord edema was evaluated based on water content. SCII induced spinal cord edema,

215 whereas miR-9 mimic reduced spinal cord edema $(p<0.05)$ (Figure 2D).

216

217

218

219

220

221

222

223

224

225

226

227

228

229

230

231

232

233

234

\section{miR-9 mimic reduced apoptosis and neuroinflammation following SCII}

We explored effects of miR-9 mimic on apoptosis at $48 \mathrm{~h}$ after SCII. Figure 3A-B demonstrates the TUNEL results. Apoptosis rate increased following SCII $(p<0.01)$. Lower apoptosis rate was found in operated rats subjected to miR-9 mimic pretreatment compared to the SCII group $(p<0.01)$. The expression of cleaved-caspase- 3 protein increased at $48 \mathrm{~h}$ after reperfusion $(p<$ 0.01), whereas miR-9 mimic significantly attenuated this downregulation $(p<0.01)$. as presented in Figure 3C.

We assessed activation of IL- 6 and IL- $\beta$ expression by ELISAs after miR-9 mimic pretreatment. The data meant that IL-6 and IL- $\beta$ were all upregulated at $48 \mathrm{~h}$ after SCII. Intrathecal pretreatment with miR-9 mimic evidently reduced this upregulation $(p<0.01)$. The findings are presented in Figure 3D-E.

\section{Bioinformatics analysis of potential miR-9 targets}

To study the participation of miR-9, target mRNAs of miR-9 were predicted by means of databases Targetscan and miRDB. 327 potential target genes were selected for miR-9 in 2 databases (Supplemental Table 1). In addition, 4829 differentially expressed genes (DEGs) (2650 upregulated and 2179 downregulated) were attained with a $|\log 2 \mathrm{FC}| \geq 1$ and $p<0.05$ between sham-operated samples and SCII samples in GSE138966 were obtained (Supplemental Table 2). A clustered heatmap of top 50 upregulated and downregulated DEGs in GSE138966 is displayed in Figure 4A. 87 intersection genes of the up-regulated DEGs and the above predicted 
235

236

237

238

239

240

241

242

243

244

245

246

247

248

249

250

251

252

253

254

255

256

257

258

259

260

261

genes were identified as potential miR-9 target genes (Supplemental Table 3 and Figure 4B). We further conducted GO and KEGG pathway analysis to detect the key target genes of miR-9.

GO analysis was performed to investigate the enrichment of miR-9 potential target genes on biological processes. Several biological processes such as "central nervous system development", "regulation of growth" and "response to cytokine" ( $p<0.05)$, were significantly enriched. The results are shown in Figure 5A. Enrichment of miR-9 potential target genes was also conducted by KEGG pathway analysis. Several significantly representative enriched pathways for potential target genes of miR-9 were identified. The results were notably enriched in several pathways, including "Notch signaling pathway", "MAPK signaling pathway", "Focal adhesion" and "Prolactin signaling pathway" $(p<0.05)$, as shown in Figure 5B and Table 1. The miRNA-pathway-gene network is demonstrated in Figure5C. It was noted that MAP2K3 and Notch2 were enriched in MAPK signaling pathway and Notch signaling pathway, respectively (supplemental Figure 1).

miR-9 mimic or SB203580 improves neurological function and alleviates the upregulation of cytokines after SCII

Figure 6A demonstrated the neurological function which was evaluated according to BBB scores. SCII induced neurological deficits of lower limbs. Compared to the SCII group, miR-9 inhibitor decreased BBB scores $(\mathrm{p}<0.01)$. SB203580 enhanced neurological function recovery $(\mathrm{p}<0.01)$. The miR-9 inhibitor + SB203580 group showed higher BBB scores $(\mathrm{p}<0.01$ vs SCII group). The results implied SB203580 could eliminate neurological deficits caused by miR-9 inhibitor. The protein expression of MAP2K3 increased at $48 \mathrm{~h}$ after SCII $(p<0.01)$, whereas miR-9 mimic notably reduced this increase $(p<0.01)$ and miR-9 inhibitor exacerbated the increase. SB203580 recuded the protein expression of MAP2K3 $(p<0.01)$ and SB203580 also reversed the effect of miR-9 inhibitor, as presented in Figure 6B. Next, we detected the expression levels of IL-6 and IL-1 $\beta$ in eight groups. As shown in Figures 6C-D, miR-9 inhibitor increased IL-6 and IL-1 $\beta$ (all $p<0.01$ vs SCII group), while SB203580 reduced the release of 
262

263

264

265

266

267

268

269

270

271

272

273

274

275

276

277

278

279

280

281

282

283

284

285

286

287

288

IL-6 and IL-1 $\beta$ (all $p<0.01$ vs SCII group). In addition, IL-6 and IL-1 $\beta$ expression were reduced in the miR-9 inhibitor+ SB203580 group compared with the miR-9 inhibitor group.

miR-9 mimic or Nocth2 siRNA improves neurological function and reduces the protein expression of cleaved-caspase 3 after SCII

Figure 7A showed the neurological function which was evaluated according to BBB scores. SCII induced severe neurological deficits of lower limbs $(p<0.01)$; meanwhile, si-Notch2 relieved neurological damage induced by SCII $(p<0.01)$, while miR-9 inhibitor aggravated neurological deterioration $(p<0.05)$. And the miR-9 inhibitor + si-Notch2 showed higher BBB scores $(p<$ 0.01 vs SCII group) indicating si-Notch2 could eliminate the damage caused by miR-9 inhibitor. The effect of miR-9 on Notch2 protein expression was similar to that on MAP2K3 protein expression, as present in Figure 7B-C. Then we assessed the expression of cleaved-caspase-3 in SCII. As shown in Figures 7B-D, intrathecal injection of Notch2 siRNA reduced the increase of cleaved-caspase-3 (vs SCII group; $p<0.01$ ). In contrast, miR-9 inhibitor increased cleavedcaspase-3 (vs SCII group; $p<0.01$ ), while cleaved-caspase-3 expression were reduced in the miR-9 inhibitor+ Notch2 siRNA group, and were comparable in the SCII and miR-9 NC groups. Discussion

The present study indicated that miR-9 mimic preserved neurological function after SCII. Candidate target genes of miR-9 were collected based on bioinformatics analysis. We further found that MAP2K3 and Notch2 protein expression were reduced by miR-9 mimic. Moreover, we demonstrated that miR-9 mimic attenuated BSCB leakage, reduced apoptosis and reduced the expression of IL-6 and IL- $\beta$ after SCII. Therefore, these results suggested that miR-9 mimic reduced neurological function injury after SCII and inhibited apoptosis and neuroinflammation possibly through MAP2K3 or Notch2-mediated signaling pathway in SCII.

The etiology of SCII is multifactorial and it is initiated through induction of primary and secondary injury. SCII, which might bring the risk of paralysis and paraplegia, has been reported in the literature but continues to be a rare presentation. Several SCII case reports have been 
289

290

291

292

293

294

295

296

297

298

299

300

301

302

303

304

305

306

307

308

309

310

311

312

313

314

315

recognized and reported(Mathkour et al. 2020; Nagano et al. 2009; Wiginton et al. 2019). James G Wiginton. etal reported a case of posterior cervical decompression leading to complete albeit transient quadriplegia caused by SCII(Wiginton et al. 2019). SCII related paraplegia caused by acute type B aortic dissection also be reported. Many pathological processes including apoptosis, microglia activation, BSCB disruption, mitophagy, oxidative stress, inflammatory reactions and autophagy play important roles in the evolution of SCII (Ha Sen Ta et al. 2019; Li et al. 2018; Li et al. 2017; Liu et al. 2017; Zhou et al. 2018). miRNAs are one important kind of noncoding RNAs and have emerged as novel targets for mediating numerous neurological diseases and regulating several physiological functions ( $\mathrm{Li}$ et al. 2019). Studies have shown that miRNAs are differentially expressed in injured spinal cord tissues after SCII and implicate their important regulatory roles in $\mathrm{SCII}(\mathrm{Hu}$ et al. 2013; Li et al. 2016). We have found miR-186-5p mimic significantly reduced neuroinflammation following SCII partly though reducing the induction of CXCL13, TLR3 or wnt5a (Chen et al. 2020a). miR-199a-5p could alleviate SCII-induced apoptosis via targeting of ECE1(Bao et al. 2018). Upregulation of miR-129-5p alleviated SCII by reducing inflammation-related BCSB and neuronal damage by inhibiting TLR3 and HMGB1associated cytokines( $\mathrm{Li}$ et al. 2017). The molecular mechanisms of miRNAs causing SCII remains largely elusive because of their complex regulatory network in SCII.

miR-9 is highly expressed in CNS and was dysregulated in different neurodegenerative disease, such as Alzheimer's disease, Huntington's disease and stroke(Lukiw 2007; Packer et al. 2008; Wei et al. 2016). miR-9 alleviated ischemic injuries by reducing anti-cardiomyocyte apoptotic affects via targeting KLF5(Yang et al. 2019). Overexpression of miR-9 alleviated ischemia injury induced by middle cerebral artery occlusion and regulated the process of autophagy by targeting ATG5 expression(Wang et al. 2018). Upregulation of miR-9 ameliorated NLRP1 inflammasome-mediated ischemic injury in rats following ischemic stroke(Cao et al. 2020). In our study, we found that miR-9 levels apparently decreased after SCII and miR-9 mimic improved neurological function following SCII. However, a previous study showed that miR-9 expression was upregulated in the spinal cord of the amyotrophic lateral sclerosis (ALS) 
316

317

318

319

320

321

322

323

324

325

326

327

328

329

330

331

332

333

334

335

336

337

338

339

340

341

342

transgenic mice(Zhou et al. 2013). The difference of expression trend may be due to the fact that miR-9 regulates neurogenesis by acting on neural or non-neural cell lineages with different model systems. In the present study, miR-9 might function through neurons. miR-9 might be associated with the proliferation and differentiation of neural stem cells (NSCs) and neural progenitor cells (NPCs) in ALS.In this study, GO analysis was performed to investigate the enrichment of miR-9 potential target genes on biological processes. Several biological processes such as "central nervous system development", "regulation of growth" and "response to cytokine" ( $p<0.05)$, were significantly enriched. KEGG pathway analysis implied several significantly representative enriched pathways for potential target genes of miR-9 were identified, such as MAPK and Notch pathways. It was noted that MAP2K3 and Notch2 were enriched in MAPK signaling pathway and Notch signaling pathway, respectively. MAPK signaling pathway and Notch signaling pathway have been studied in CNS. miR-21 exerted its protective effect against blood brain barrier (BBB) disruption by blocking the MAPK signaling pathway via targeted inhibition of MAP2K3(Yao et al. 2018). Neurotrophin-3 inhibited the content of TNF- $\beta$, IL-6 and IL-1 $\beta$ in spinal cord injury through inhibiting the MAPK signaling pathway (Ye et al. 2020). Metformin reduced microglial activation and inhibited the production of pro-inflammatory cytokines including IL-6, IL-1 $\beta$ and TNF- $\alpha$ via MAPK and NF- $\kappa B$ signaling pathway to improve neurobehavioral function following traumatic brain injury(Tao et al. 2018). Inhibition of Notch2 reduced cerebral I/R-induced cell death in the short term(Meng et al. 2015). miR-485-5p inhibited neuron apoptosis following I/R injury via targeting Rac1/Notch2 signaling (Chen et al. 2020). In our study, we found that miR-9 mimic markedly attenuated this upregulation of MAP2K3 and Notch2. Moreover, miR-9 mimic inhibited the increase of IL-6 and IL- $\beta$ induced by SCII. Furthermore, miR-9 mimic reduced the expression of cleavedcaspase-3 and apoptosis rate after SCII. In addition, miR-9 inhibitor increased IL-6 and IL-1 $\beta$ after SCII, while SB203580 reduced the upregulation of IL-6 and IL-1 $\beta$. IL-6 and IL-1 $\beta$ expression were reduced in the miR-9 inhibitor+ SB203580 group compared to the miR-9 inhibitor group. Notch2 siRNA reduced the increase of cleaved-caspase-3 after SCII. miR-9 
343 inhibitor increased cleaved-caspase-3, while cleaved-caspase-3 expression were reduced in the 344 miR-9 inhibitor+ Notch2 siRNA group. MAP2K3 might be the target gene of miR-9 for 345 involving neuroinflammation after SCII. Notch2 might be the target gene of miR-9 for regulating 346 apoptosis after SCII.

347 The limitation of the present study is that we only explored the expression of miR-9 during $72 \mathrm{~h}$ 348 following SCII. We found that miR-9 levels significantly decreased at 24 and $48 \mathrm{~h}$. At $48 \mathrm{~h}$ after 349 SCII, miR-9 expression demonstrated the lowest level. The expression level of miR-9 returned to 350 the Sham group level at $72 \mathrm{~h}$ after SCII. Longer time course is needed to detect for further exploring the roles of miR-9 after $72 \mathrm{~h}$ in SCII.

352

In conclusion, miR-9 mimic preserved hind limb function after SCII through reducing apoptosis and neuroinflammation. And miR-9 mimic might protect against SCII though MAP2K3mediated neuroinflammation and Notch2-mediated apoptosis.

\section{Conflict of interest}

The authors declare that they have no conflicts of interest.

\section{Reference}

Altintas O, Ozgen Altintas M, Kumas M, and Asil T. 2016. Neuroprotective effect of ischemic preconditioning via modulating the expression of cerebral miRNAs against transient cerebral ischemia in diabetic rats. Neurol Res 38:1003-1011. 10.1080/01616412.2016.1232013

Bao N, Fang B, Lv H, Jiang Y, Chen F, Wang Z, and Ma H. 2018. Upregulation of miR-199a-5p Protects Spinal Cord Against Ischemia/Reperfusion-Induced Injury via Downregulation of ECE1 in Rat. Cell Mol Neurobiol 38:1293-1303. 10.1007/s10571-018-0597-2

Basso DM, Beattie MS, and Bresnahan JC. 1995. A sensitive and reliable locomotor rating scale for open field testing in rats. $J$ Neurotrauma 12:1-21. 10.1089/neu.1995.12.1

Cao Y, Zhang H, Lu X, Wang J, Zhang X, Sun S, Bao Z, Tian W, Ning S, Wang L, and Cui L. 2020. Overexpression of MicroRNA-9a-5p Ameliorates NLRP1 Inflammasome-mediated Ischemic Injury in Rats Following Ischemic Stroke. Neuroscience 444:106-117. 10.1016/j.neuroscience.2020.01.008

Chen F, Li X, Li Z, Qiang Z, and Ma H. 2020a. Altered expression of MiR-186-5p and its target genes after spinal cord ischemia-reperfusion injury in rats. Neurosci Lett 718:134669. 10.1016/j.neulet.2019.134669

Chen F, Li X, Li Z, Zhou Y, Qiang Z, and Ma H. 2020b. The roles of chemokine (C-X-C motif) ligand 13 in spinal cord ischemia-reperfusion injury in rats. Brain Res 1727:146489. 10.1016/j.brainres.2019.146489 
374

375

376

377

378

379

380

381

382

383

384

385

386

387

388

389

390

391

392

393

394

395

396

397

398

399

400

401

402

403

404

405

406

407

408

409

410

411

412

413

414

Chen S, Wang M, Yang H, Mao L, He Q, Jin H, Ye ZM, Luo XY, Xia YP, and Hu B. 2017. LncRNA TUG1 sponges microRNA-9 to promote neurons apoptosis by up-regulated Bcl2111 under ischemia. Biochem Biophys Res Commun 485:167-173. 10.1016/j.bbrc.2017.02.043

Chen X, Zhang S, Shi P, Su Y, Zhang D, and Li N. 2020c. MiR-485-5p promotes neuron survival through mediating Rac1/Notch2 signaling pathway after cerebral ischemia/reperfusion. Curr Neurovasc Res. $10.2174 / 1567202617666200415154822$

Chi W, Meng F, Li Y, Li P, Wang G, Cheng H, Han S, and Li J. 2014. Impact of microRNA-134 on neural cell survival against ischemic injury in primary cultured neuronal cells and mouse brain with ischemic stroke by targeting HSPA12B. Brain Res 1592:22-33. 10.1016/j.brainres.2014.09.072

Ding L, Fu WJ, Di HY, Zhang XM, Lei YT, Chen KZ, Wang T, and Wu HF. 2020. Expression of long non-coding RNAs in complete transection spinal cord injury: a transcriptomic analysis. Neural Regen Res 15:15601567. $10.4103 / 1673-5374.274348$

Ege E, Ilhan A, Gurel A, Akyol O, and Ozen S. 2004. Erdosteine ameliorates neurological outcome and oxidative stress due to ischemia/reperfusion injury in rabbit spinal cord. Eur J Vasc Endovasc Surg 28:379-386. 10.1016/j.ejvs.2004.06.004

Fang B, Li XQ, Bi B, Tan WF, Liu G, Zhang Y, and Ma H. 2015. Dexmedetomidine attenuates blood-spinal cord barrier disruption induced by spinal cord ischemia reperfusion injury in rats. Cell Physiol Biochem 36:373383. 10.1159/000430107

Gao HY, Huo FC, Wang HY, and Pei DS. 2017. MicroRNA-9 inhibits the gastric cancer cell proliferation by targeting TNFAIP8. Cell Prolif 50. 10.1111/cpr.12331

Goldim MPS, Della Giustina A, and Petronilho F. 2019. Using Evans Blue Dye to Determine Blood-Brain Barrier Integrity in Rodents. Curr Protoc Immunol 126:e83. 10.1002/cpim.83

Ha Sen Ta N, Nuo M, Meng QT, and Xia ZY. 2019. The Pathway of Let-7a-1/2-3p and HMGB1 Mediated Dexmedetomidine Inhibiting Microglia Activation in Spinal Cord Ischemia-Reperfusion Injury Mice. $J$ Mol Neurosci 69:106-114. 10.1007/s12031-019-01338-4

Hawley ZCE, Campos-Melo D, and Strong MJ. 2019. MiR-105 and miR-9 regulate the mRNA stability of neuronal intermediate filaments. Implications for the pathogenesis of amyotrophic lateral sclerosis (ALS). Brain Res 1706:93-100. 10.1016/j.brainres.2018.10.032

He F, Shi E, Yan L, Li J, and Jiang X. 2015. Inhibition of micro-ribonucleic acid-320 attenuates neurologic injuries after spinal cord ischemia. J Thorac Cardiovasc Surg 150:398-406. 10.1016/j.jtcvs.2015.03.066

Hu JR, Lv GH, and Yin BL. 2013. Altered microRNA expression in the ischemic-reperfusion spinal cord with atorvastatin therapy. J Pharmacol Sci 121:343-346. 10.1254/jphs.12235sc

Huang da W, Sherman BT, and Lempicki RA. 2009. Systematic and integrative analysis of large gene lists using DAVID bioinformatics resources. Nat Protoc 4:44-57. 10.1038/nprot.2008.211

Huang W, Liu X, Cao J, Meng F, Li M, Chen B, and Zhang J. 2015. miR-134 regulates ischemia/reperfusion injuryinduced neuronal cell death by regulating CREB signaling. J Mol Neurosci 55:821-829. 10.1007/s12031014-0434-0

Li JA, Zan CF, Xia P, Zheng CJ, Qi ZP, Li CX, Liu ZG, Hou TT, and Yang XY. 2016a. Key genes expressed in different stages of spinal cord ischemia/reperfusion injury. Neural Regen Res 11:1824-1829. 10.4103/16735374.194754

Li L, Jiang HK, Li YP, and Guo YP. 2015a. Hydrogen sulfide protects spinal cord and induces autophagy via miR-

PeerJ reviewing PDF | (2020:12:56677:1:1:NEW 15 Apr 2021) 
415

416

417

418

419

420

421

422

423

424

425

426

427

428

429

430

431

432

433

434

435

436

437

438

439

440

441

442

443

444

445

446

447

448

449

450

451

452

453

454

455

30c in a rat model of spinal cord ischemia-reperfusion injury. J Biomed Sci 22:50. 10.1186/s12929-0150135-1

Li X, Lou X, Xu S, Wang Q, Shen M, and Miao J. 2018a. Knockdown of miR-372 Inhibits Nerve Cell Apoptosis Induced by Spinal Cord Ischemia/Reperfusion Injury via Enhancing Autophagy by Up-regulating Beclin-1. J Mol Neurosci 66:437-444. 10.1007/s12031-018-1179-y

Li XQ, Cao XZ, Wang J, Fang B, Tan WF, and Ma H. 2014a. Sevoflurane preconditioning ameliorates neuronal deficits by inhibiting microglial MMP-9 expression after spinal cord ischemia/reperfusion in rats. Mol Brain 7:69. 10.1186/s13041-014-0069-7

Li XQ, Chen FS, Tan WF, Fang B, Zhang ZL, and Ma H. 2017. Elevated microRNA-129-5p level ameliorates neuroinflammation and blood-spinal cord barrier damage after ischemia-reperfusion by inhibiting HMGB1 and the TLR3-cytokine pathway. J Neuroinflammation 14:205. 10.1186/s12974-017-0977-4

Li XQ, Fang B, Tan WF, Wang ZL, Sun XJ, Zhang ZL, and Ma H. 2016b. miR-320a affects spinal cord edema through negatively regulating aquaporin-1 of blood-spinal cord barrier during bimodal stage after ischemia reperfusion injury in rats. BMC Neurosci 17:10. 10.1186/s12868-016-0243-1

Li XQ, Lv HW, Wang ZL, Tan WF, Fang B, and Ma H. 2015b. MiR-27a ameliorates inflammatory damage to the blood-spinal cord barrier after spinal cord ischemia: reperfusion injury in rats by downregulating TICAM-2 of the TLR4 signaling pathway. J Neuroinflammation 12:25. 10.1186/s12974-015-0246-3

Li XQ, Wang J, Fang B, Tan WF, and Ma H. 2014b. Intrathecal antagonism of microglial TLR4 reduces inflammatory damage to blood-spinal cord barrier following ischemia/reperfusion injury in rats. Mol Brain 7:28. 10.1186/1756-6606-7-28

Li XQ, Yu Q, Chen FS, Tan WF, Zhang ZL, and Ma H. 2018b. Inhibiting aberrant p53-PUMA feedback loop activation attenuates ischaemia reperfusion-induced neuroapoptosis and neuroinflammation in rats by downregulating caspase 3 and the NF-кB cytokine pathway. J Neuroinflammation 15:250. 10.1186/s12974018-1271-9

Li XQ, Yu Q, Tan WF, Zhang ZL, and Ma H. 2018c. MicroRNA-125b mimic inhibits ischemia reperfusion-induced neuroinflammation and aberrant p53 apoptotic signalling activation through targeting TP53INP1. Brain Behav Immun 74:154-165. 10.1016/j.bbi.2018.09.002

Li Z, Han K, Zhang D, Chen J, Xu Z, and Hou L. 2019. The role of long noncoding RNA in traumatic brain injury. Neuropsychiatr Dis Treat 15:1671-1677. 10.2147/ndt.S206624

Liu K, Yan L, Jiang X, Yu Y, Liu H, Gu T, and Shi E. 2017. Acquired inhibition of microRNA-124 protects against spinal cord ischemia-reperfusion injury partially through a mitophagy-dependent pathway. $J$ Thorac Cardiovasc Surg 154:1498-1508. 10.1016/j.jtcvs.2017.05.046

Liu ZG, Li Y, Jiao JH, Long H, Xin ZY, and Yang XY. 2020. MicroRNA regulatory pattern in spinal cord ischemiareperfusion injury. Neural Regen Res 15:2123-2130. 10.4103/1673-5374.280323

Lukiw WJ. 2007. Micro-RNA speciation in fetal, adult and Alzheimer's disease hippocampus. Neuroreport 18:297300. 10.1097/WNR.0b013e3280148e8b

Mathkour M, Werner C, Riffle J, Scullen T, Dallapiazza RF, Dumont A, and Maulucci C. 2020. Reperfusion "White Cord" Syndrome in Cervical Spondylotic Myelopathy: Does Mean Arterial Pressure Goal Make a Difference? Additional Case and Literature Review. World Neurosurg 137:194-199. 10.1016/j.wneu.2020.01.062

Meng S, Su Z, Liu Z, Wang N, and Wang Z. 2015. Rac1 contributes to cerebral ischemia reperfusion-induced injury 
in mice by regulation of Notch2. Neuroscience 306:100-114. 10.1016/j.neuroscience.2015.08.014

Nagano N, Kikuchi K, Amano A, and Yamaoka H. 2009. Should we consider surgical intervention for spinal cord ischemia due to acute type B aortic dissection? Eur $J$ Cardiothorac Surg 35:547-549. 10.1016/j.ejcts.2008.12.014

Packer AN, Xing Y, Harper SQ, Jones L, and Davidson BL. 2008. The bifunctional microRNA miR-9/miR-9* regulates REST and CoREST and is downregulated in Huntington's disease. $J$ Neurosci 28:14341-14346. 10.1523/jneurosci.2390-08.2008

Tao L, Li D, Liu H, Jiang F, Xu Y, Cao Y, Gao R, and Chen G. 2018. Neuroprotective effects of metformin on traumatic brain injury in rats associated with NF- $\mathrm{kB}$ and MAPK signaling pathway. Brain Res Bull 140:154-161. 10.1016/j.brainresbull.2018.04.008

Wang D, Chen F, Fang B, Zhang Z, Dong Y, Tong X, and Ma H. 2020. MiR-128-3p Alleviates Spinal Cord Ischemia/Reperfusion Injury Associated Neuroinflammation and Cellular Apoptosis via SP1 Suppression in Rat. Front Neurosci 14:609613. 10.3389/fnins.2020.609613

Wang H, Zhang W, Zuo Y, Ding M, Ke C, Yan R, Zhan H, Liu J, and Wang J. 2015. miR-9 promotes cell proliferation and inhibits apoptosis by targeting LASS2 in bladder cancer. Tumour Biol 36:9631-9640. 10.1007/s13277-015-3713-7

Wang J, Peng R, Zhang Z, Zhang Y, Dai Y, and Sun Y. 2021. Identification and Validation of Key Genes in Hepatocellular Carcinoma by Bioinformatics Analysis. Biomed Res Int 2021:6662114. $10.1155 / 2021 / 6662114$

Wang N, Yang L, Zhang H, Lu X, Wang J, Cao Y, Chen L, Wang X, Cong L, Li J, Wang N, Liu Z, and Wang L. 2018. MicroRNA-9a-5p Alleviates Ischemia Injury After Focal Cerebral Ischemia of the Rat by Targeting ATG5-Mediated Autophagy. Cell Physiol Biochem 45:78-87. 10.1159/000486224

Wei N, Xiao L, Xue R, Zhang D, Zhou J, Ren H, Guo S, and Xu J. 2016. MicroRNA-9 Mediates the Cell Apoptosis by Targeting Bcl2111 in Ischemic Stroke. Mol Neurobiol 53:6809-6817. 10.1007/s12035-015-9605-4

Wiginton JGt, Brazdzionis J, Mohrdar C, Sweiss R, and Lawandy S. 2019. Spinal Cord Reperfusion Injury: Case Report, Review of the Literature, and Future Treatment Strategies. Cureus 11:e5279. 10.7759/cureus.5279

$\mathrm{Xu} \mathrm{Z}$, and Li Z. 2020. Experimental Study on the Role of Apelin-13 in Alleviating Spinal Cord Ischemia Reperfusion Injury Through Suppressing Autophagy. Drug Des Devel Ther 14:1571-1581. $10.2147 /$ dddt.S241066

Yang D, Yu J, Liu HB, Yan XQ, Hu J, Yu Y, Guo J, Yuan Y, and Du ZM. 2019. The long non-coding RNA TUG1miR-9a-5p axis contributes to ischemic injuries by promoting cardiomyocyte apoptosis via targeting KLF5. Cell Death Dis 10:908. 10.1038/s41419-019-2138-4

Yao K, Yang Q, Li Y, Lan T, Yu H, and Yu Y. 2020. MicroRNA-9 mediated the protective effect of ferulic acid on hypoxic-ischemic brain damage in neonatal rats. PLoS One 15:e0228825. 10.1371/journal.pone.0228825

Yao X, Wang Y, and Zhang D. 2018. microRNA-21 Confers Neuroprotection Against Cerebral IschemiaReperfusion Injury and Alleviates Blood-Brain Barrier Disruption in Rats via the MAPK Signaling Pathway. J Mol Neurosci 65:43-53. 10.1007/s12031-018-1067-5

Ye J, Xue R, Ji ZY, Zou CJ, Chen YQ, Wang JJ, and Cheng XD. 2020. Effect of NT-3 on repair of spinal cord injury through the MAPK signaling pathway. Eur Rev Med Pharmacol Sci 24:2165-2172. 10.26355/eurrev_202003_20481

Zhou F, Guan Y, Chen Y, Zhang C, Yu L, Gao H, Du H, Liu B, and Wang X. 2013a. miRNA-9 expression is 
upregulated in the spinal cord of G93A-SOD1 transgenic mice. Int J Clin Exp Pathol 6:1826-1838.

Zhou J, Lin W, Chen H, Fan Y, and Yang C. 2016. TRESK contributes to pain threshold changes by mediating apoptosis via MAPK pathway in the spinal cord. Neuroscience 339:622-633. 10.1016/j.neuroscience.2016.10.039

Zhou XM, Liu J, Wang Y, and Zhang MH. 2018. Silencing of long noncoding RNA MEG3 enhances cerebral protection of dexmedetomidine against hypoxic-ischemic brain damage in neonatal mice by binding to miR-129-5p. J Cell Biochem. 10.1002/jcb.28075

Zhou YF, Li L, Feng F, Yuan H, Gao DK, Fu LA, and Fei Z. 2013b. Osthole attenuates spinal cord ischemiareperfusion injury through mitochondrial biogenesis-independent inhibition of mitochondrial dysfunction in rats. J Surg Res 185:805-814. 10.1016/j.jss.2013.06.044

Zhu M, Xu Y, Ge M, Gui Z, and Yan F. 2015. Regulation of UHRF1 by microRNA-9 modulates colorectal cancer cell proliferation and apoptosis. Cancer Sci 106:833-839. 10.1111/cas.12689

\section{Fig legends}

Figure 1. miRNA expression of miR-9 after SCII. (A) miR-9 relative expression at $24 \mathrm{~h}, 48$ $\mathrm{h}$, and $72 \mathrm{~h}$ after SCII $(\mathrm{n}=3) .{ }^{*} p<0.05,{ }^{* *} p<0.01$, versus group sham, ${ }^{\&} p<0.05,{ }^{\&} \& p<0.01$, versus group SCII 48h. (B) miR-9 relative expression in four groups at $48 \mathrm{~h}$ after SCII $(\mathrm{n}=3)$. (C) Neurological function was evaluated by means of the BBB scoring at $48 \mathrm{~h}$ after SCII $(\mathrm{n}=8)$. ${ }^{\#} p<0.05,{ }^{\#} p<0.01$, versus group sham, ${ }^{\circledR} p<0.05$, ${ }^{\circledR}{ }^{\circledR} p<0.01$, versus group SCII, for (B) and (C).

Figure 2. A clustered heatmap of top 50 upregulated and downregulated DEGs in GSE138966 (A). Blue color signifies low expression; red color signifies high expression. Venn diagrams of potential miR-9 target genes (B).

Figure 3. Effects of on BSCB disruption and spinal cord water content at $48 \mathrm{~h}$ after SCII. (A) Representative fluorescence of extravasating EB in four groups $(n=3)$. scale bar $=500 \mu \mathrm{m}$. (B) EB fluorescence density $\left(\mathrm{INT} / \mathrm{mm}^{2}\right)(\mathrm{n}=3)$. (C) EB content $(\mu \mathrm{g} / \mathrm{g})$ of spinal cord $(\mathrm{n}=3)$. (D) Percentage water content $(\mathrm{n}=3) .{ }^{\#} p<0.05,{ }^{\# \#} p<0.01$, versus group sham, ${ }^{@} p<0.05$, ${ }^{@}{ }^{0} p<$ 0.01 , versus group SCII.

Figure 4. miR-9 mimic reduced apoptosis rate and the production of IL-6 and IL-1 $\beta$ at $48 \mathrm{~h}$ after 
527 SCII. (A) Representative TUNEL stain in four groups $(\mathrm{n}=3)$. scale bar $=20 \mu \mathrm{m}$. (B) Apoptosis 528 rate in four groups. (C) Effects of miR-9 mimic on the expression of cleaved caspase-3 $(\mathrm{n}=3)$. 529 (D-E) IL-6 and IL-1 $\beta$ at $48 \mathrm{~h}$ after SCII $(\mathrm{n}=3) .{ }^{\#} p<0.05,{ }^{\#} p<0.01$, versus group sham, ${ }^{\circledR} p<$ $5300.05, @ @ p 0.01$, versus group SCII.

531 Figure 5. Bioinformatics analysis of potential rno-miR-9 target mRNAs. The GO annotations for 532 biological process and KEGG pathway analysis of significant GO (A) and KEGG (B)

533 enrichment terms. (C) The miR-pathway-gene network.

Figure 6. Intrathecal pretreatment with miR-9 mimic or SB203580 ameliorates neurological function and alleviates the upregulation of cytokines after SCII. (A) Neurological function scores at $48 \mathrm{~h}$ after SCII in eight groups. Each symbol represents one rat $(\mathrm{n}=8)$. (B) The protein expression of MAP2K3 at $48 \mathrm{~h}$ after SCII in eight groups $(\mathrm{n}=3)$. (C-D) Measurements of IL-6 and IL-1 $\beta$ expression levels by ELISA in eight groups $(\mathrm{n}=3)$. ${ }^{\#} p<0.05$, ${ }^{\#} p<0.01$, versus group sham. @ $p<0.05$, @ $p<0.01$, versus group SCII.

Figure 7. Intrathecal pretreatment with miR-9 mimic or Notch2 siRNA ameliorates neurological function and reduces the increase of cleaved-caspase3 after SCII. (A) Neurological function scores at $48 \mathrm{~h}$ after SCII in eight groups. Each symbol represents one rat $(\mathrm{n}=8)$. (B-D) Representative Western blotting and the protein expression of Notch2 and cleaved-caspase-3 at $48 \mathrm{~h}$ after SCII in eight groups $(\mathrm{n}=3) .{ }^{\#} p<0.05,{ }^{\#} p<0.01$, versus group sham. ${ }^{\circledR} p<0.05$, ${ }^{@} p$ $<0.01$, versus group SCII.

Table 1. KEGG Pathway Terms for potential targets of miR-9 $(p<0.05)$ * MAP2K3 and Notch2 were enriched in MAPK signaling pathway and Notch signaling pathway, respectively.

Supplemental Table 1. 327 potential target genes were selected for miR-9 in 2 databases.

Supplemental Table 2. 4829 differentially expressed genes (DEGs) in GSE138966. 
553 Supplemental Table 3. The intersection genes of the up-regulated DEGs and the above predicted 554 genes were identified as potential miR-9 target genes.

555 Supplemental Figure 1. MAP2K3(MKK3) in MAPK signaling pathway (A) and Notch2 in Notch 556 signaling pathway (B). 
Figure 1

Figure 1. miRNA expression of miR-9 after SCll.

(A) miR-9 relative expression at $24 \mathrm{~h} \square 48 \mathrm{~h} \square$ and $72 \mathrm{~h}$ after $\mathrm{SCII}(\mathrm{n}=3) .{ }^{*} p<0.05,{ }^{* *} p<0.01$, versus group sham, ${ }^{\alpha} p<0.05,{ }^{\alpha \varepsilon} p<0.01$, versus group SCII 48h. (B) miR-9 relative expression in four groups at $48 \mathrm{~h}$ after $\mathrm{SCII}(\mathrm{n}=3)$. (C) Neurological function was evaluated by means of the BBB scoring at $48 \mathrm{~h}$ after SCII $(n=8) .{ }^{\#} p<0.05,{ }^{\# \#} p<0.01$, versus group sham, ${ }^{\circledR} p<0.05,{ }^{\circledR} p<0.01$, versus group SCII, for (B) and (C).
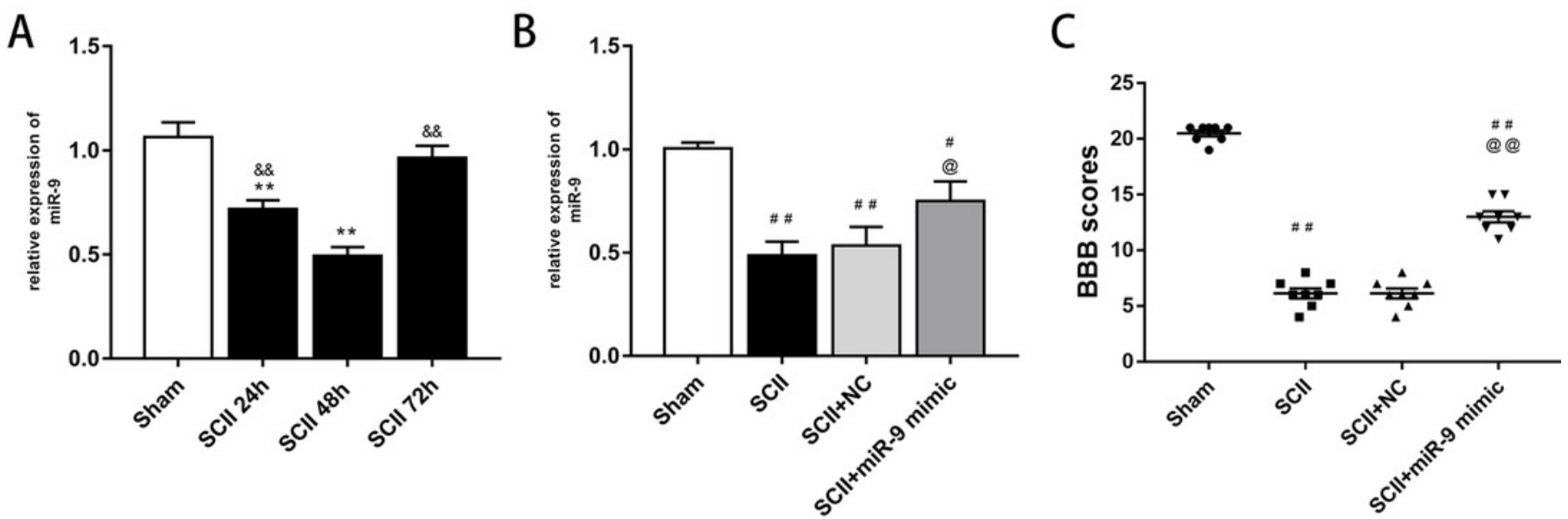


\section{Figure 2}

Figure 2. A clustered heatmap of top 50 upregulated and downregulated DEGs in GSE138966 (A). Blue color signifies low expression; red color signifies high expression. Venn diagrams of potential miR-9 target genes (B).

A clustered heatmap of top 50 upregulated and downregulated DEGs in GSE138966 (A). Blue color signifies low expression; red color signifies high expression. Venn diagrams of potential miR-9 target genes (B).

A

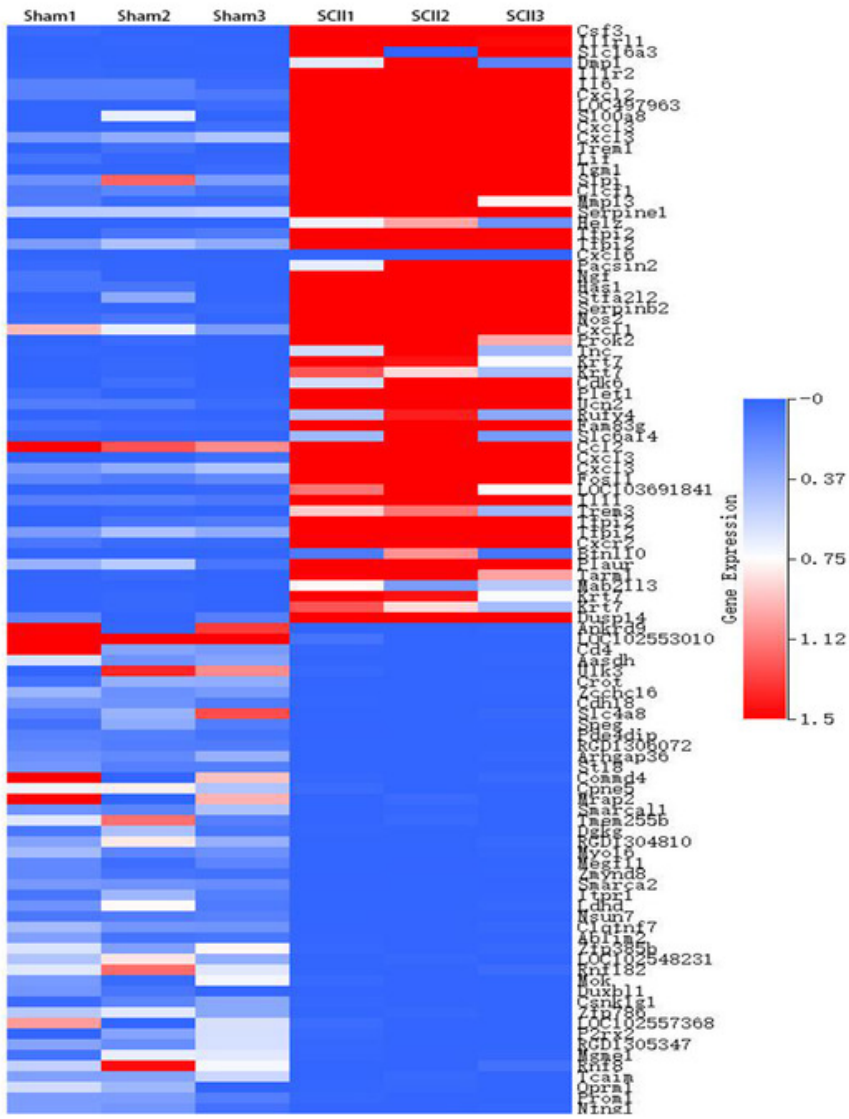

B

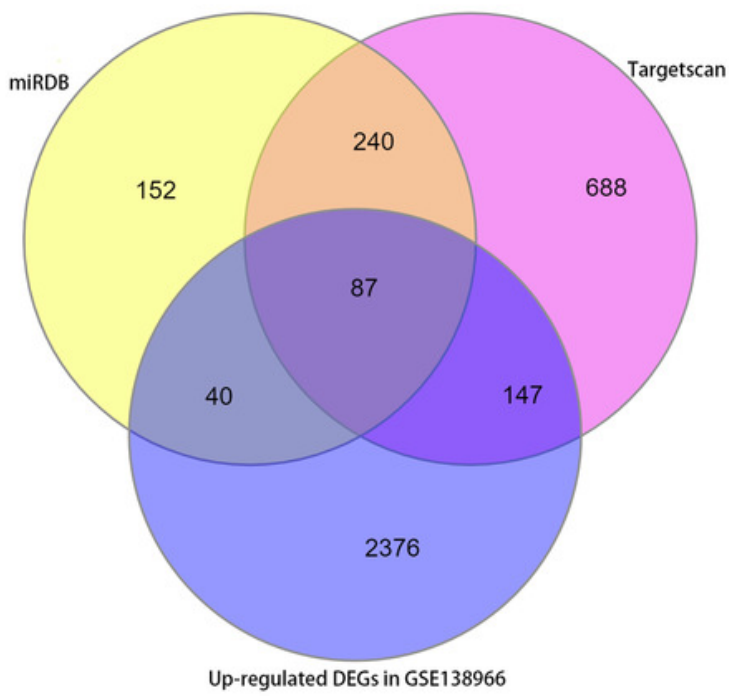




\section{Figure 3}

Figure 3. Effects of on BSCB disruption and spinal cord water content at $48 \mathrm{~h}$ after SCII.

(A) Representative fluorescence of extravasating EB in four groups $(n=3)$. scale bar $=500$ $\mu m$. (B) EB fluorescence density $\left(I N T / \mathrm{mm}^{2}\right)(n=3)$. (C) EB content $(\mu \mathrm{g} / \mathrm{g})$ of spinal cord $(n=$ 3). (D) Percentage water content $(n=3) .{ }^{\#} p<0.05,{ }^{\# \#} p<0.01$, versus group sham, ${ }^{\circledR} p<$ $0.05,{ }^{\circledR ®} p<0.01$, versus group SCII.

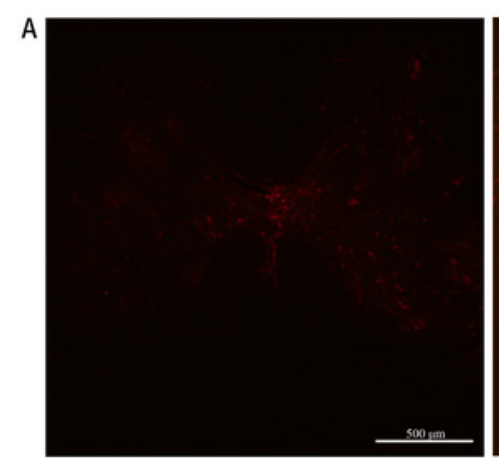

Sham

B

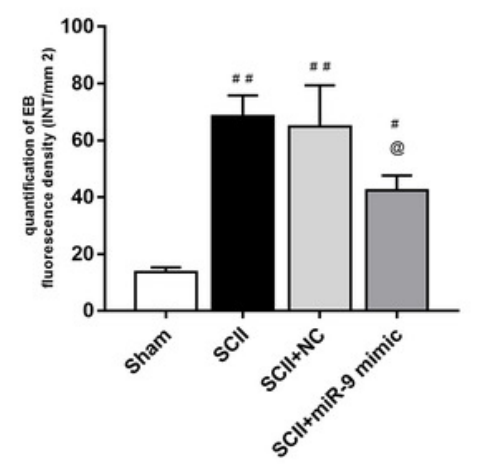

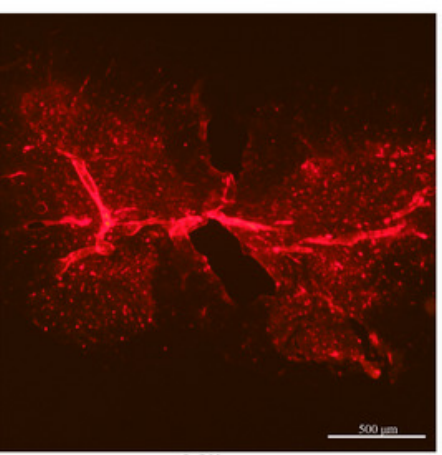

SCII

C

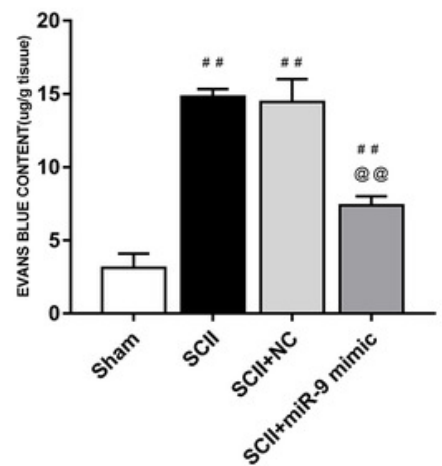

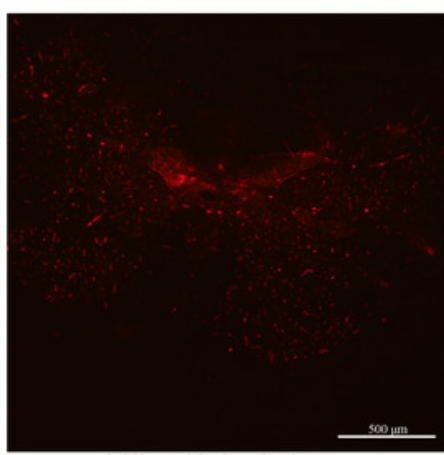

SCII+miR-9 mimic

D

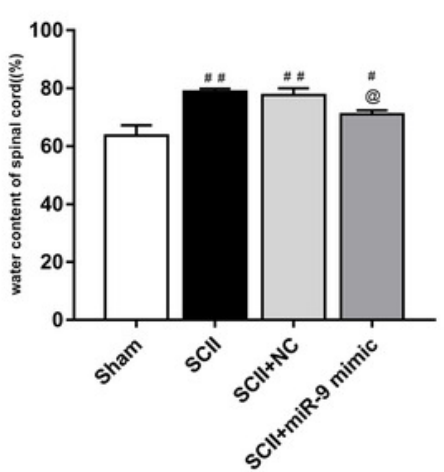


Figure 4

Figure 4. miR-9 mimic reduced apoptosis rate and the production of IL- 6 and IL-1 $1 \beta$ at 48 h after SCII.

(A) Representative TUNEL stain in four groups $(n=3)$. scale bar $=20 \mu \mathrm{m}$. (B) Apoptosis rate in four groups. (C) Effects of miR-9 mimic on the expression of cleaved caspase-3 $(n=3)$. (D-

E) IL- 6 and IL-1 $1 \beta$ at $48 \mathrm{~h}$ after SCII $(\mathrm{n}=3) .{ }^{*} p<0.05,{ }^{\# \#} p<0.01$, versus group sham, ${ }^{\circledR} p<$ $0.05,{ }^{\circledR} p<0.01$, versus group SCII.

A

A
$\because \cdots$
$\because \cdots$

B

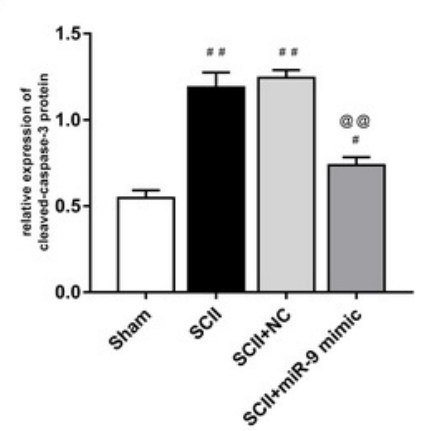

C
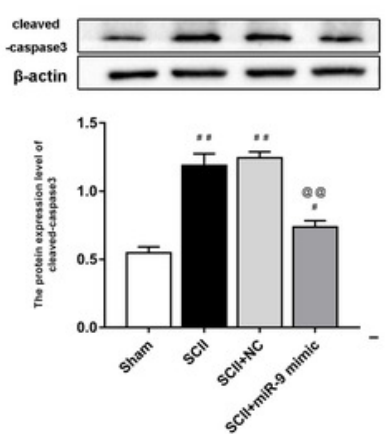

D

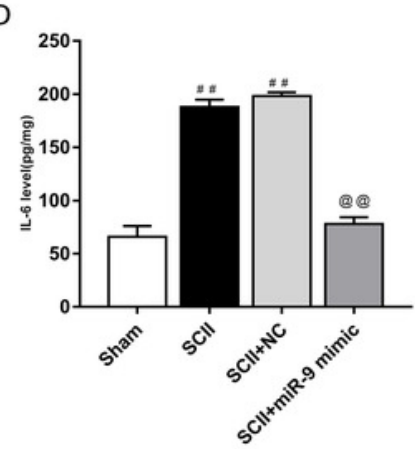

E

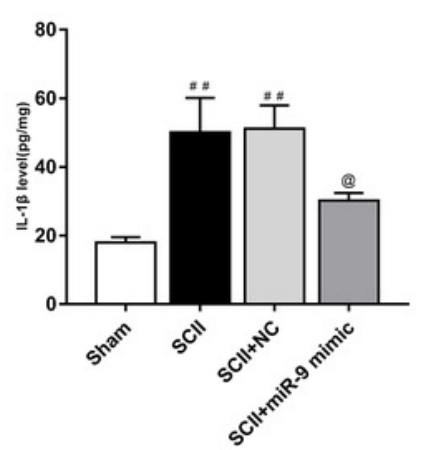




\section{Figure 5}

Figure 5. Bioinformatics analysis of potential rno-miR-9 target mRNAs.

The GO annotations for biological process and KEGG pathway analysis of significant GO (A) and KEGG (B) enrichment terms. (C) The miR-pathway-gene network. 
A

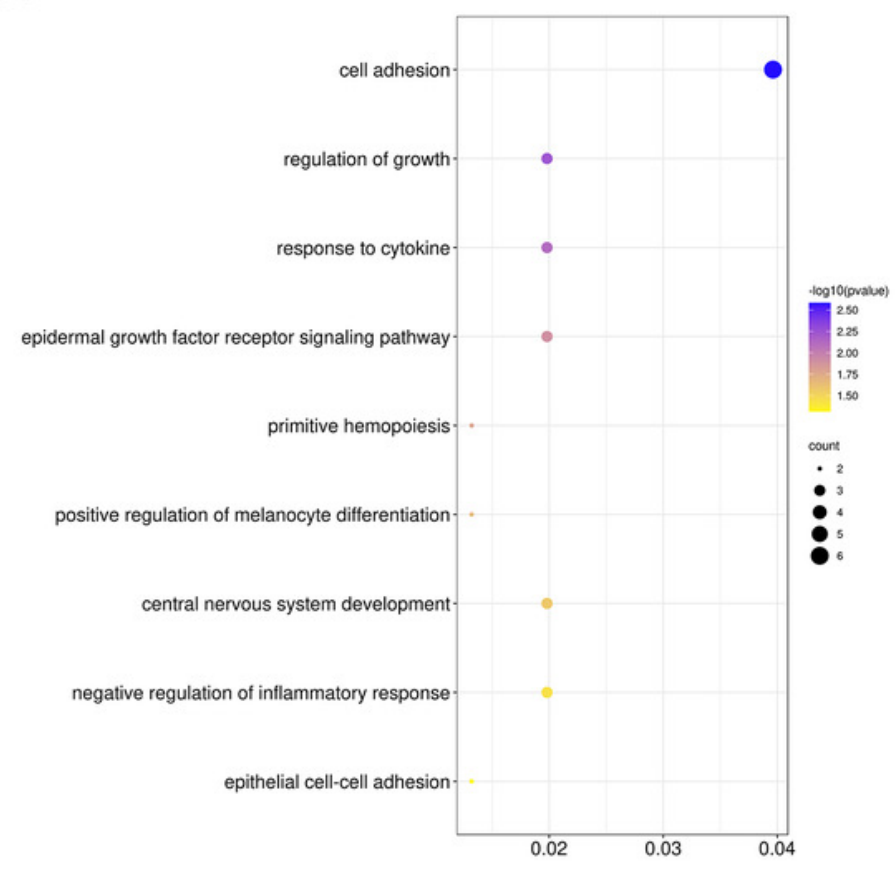

B

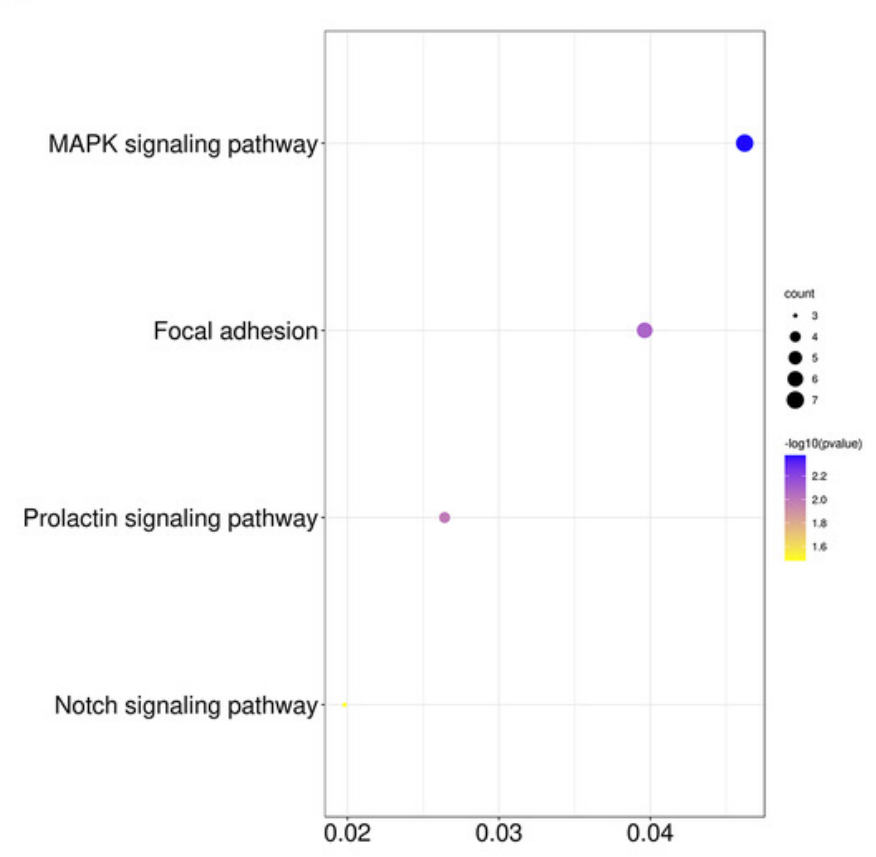

C

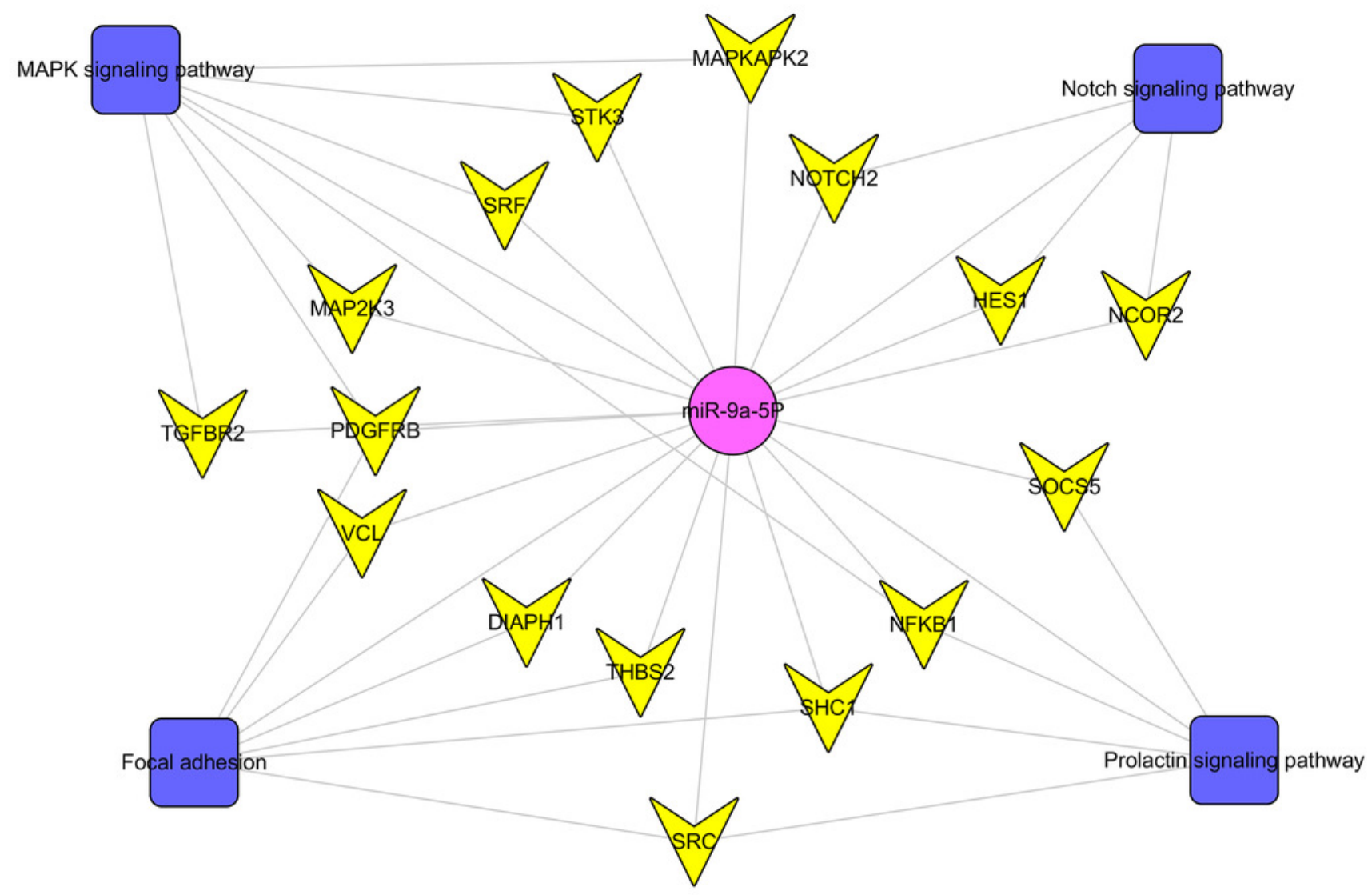




\section{Figure 6}

Figure 6. Intrathecal pretreatment with miR-9 mimic or SB203580 ameliorates neurological function and alleviates the upregulation of cytokines after SCll.

(A) Neurological function scores at $48 \mathrm{~h}$ after SCII in eight groups. Each symbol represents one rat $(n=8)$. (B) The protein expression of MAP2K3 at $48 \mathrm{~h}$ after SCII in eight groups $(n=$ 3). (C-D) Measurements of IL-6 and IL-1 $\beta$ expression levels by ELISA in eight groups $(n=3)$. ${ }^{\#} p<0.05,{ }^{\# \#} p<0.01$, versus group sham. ${ }^{\circledR} p<0.05,{ }^{\circledR} p<0.01$, versus group $\mathrm{SCll}$. 
A

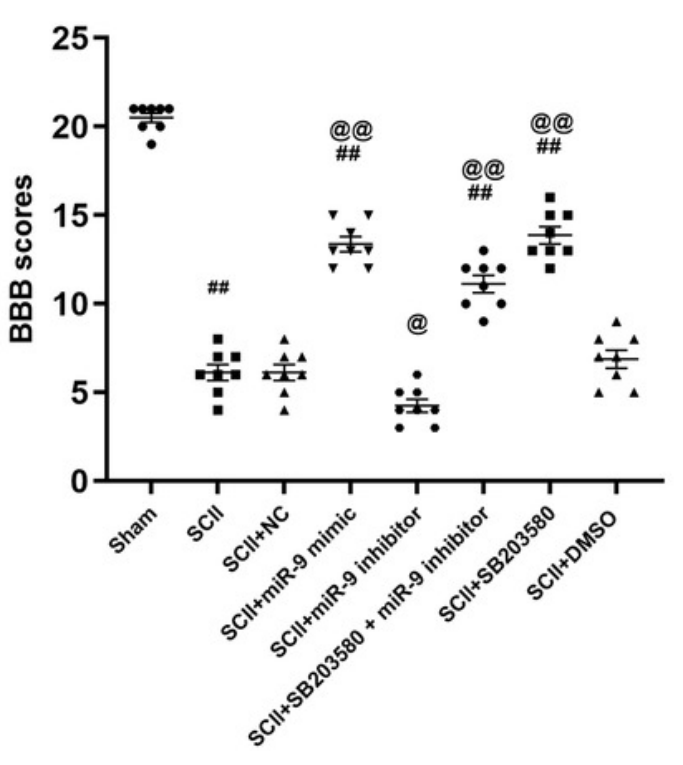

C

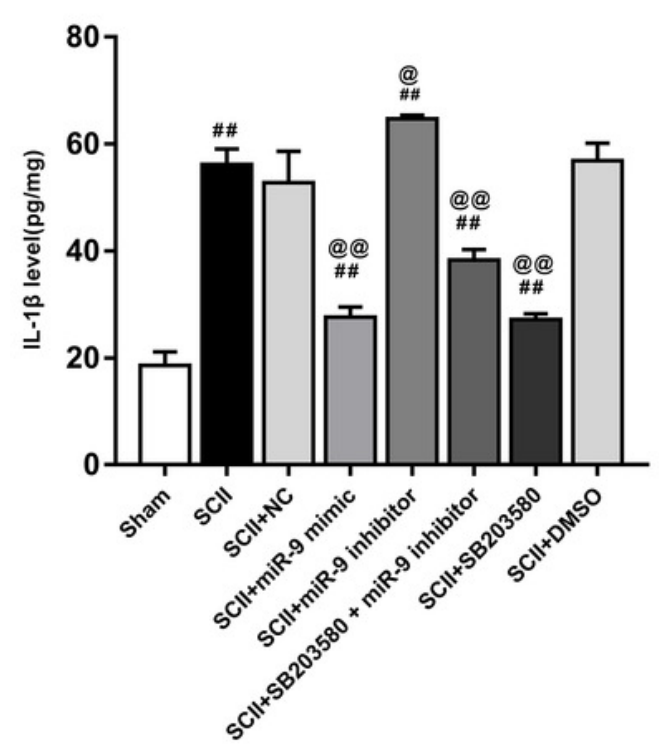

B

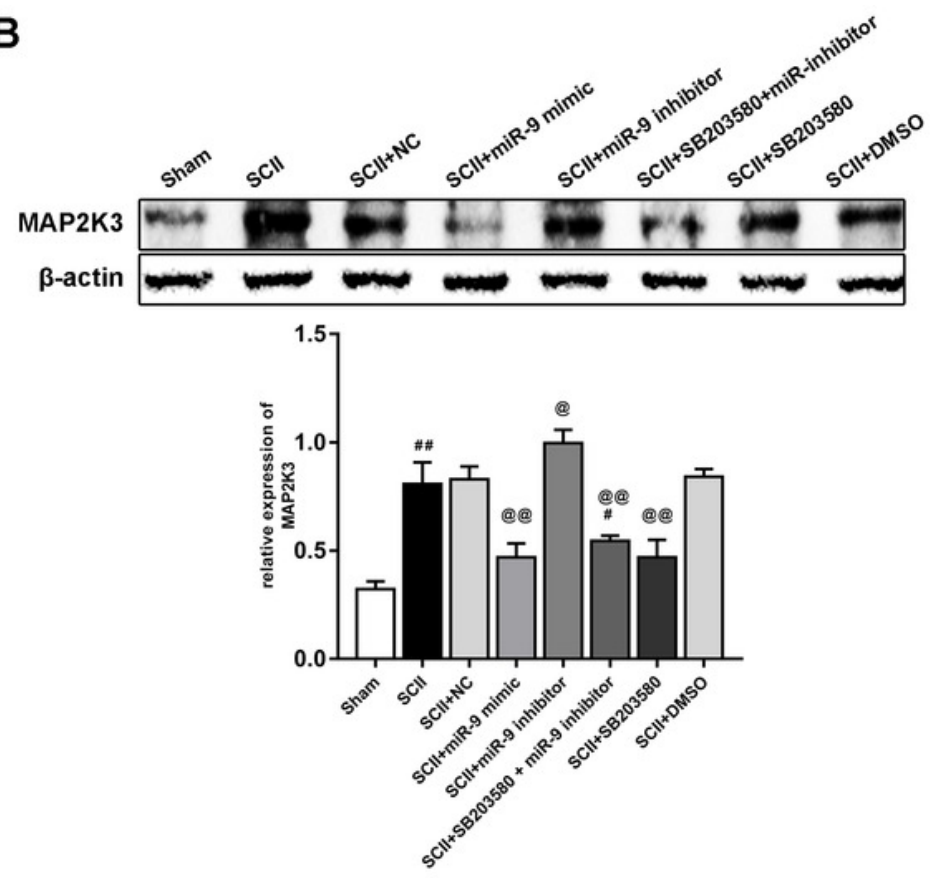

D

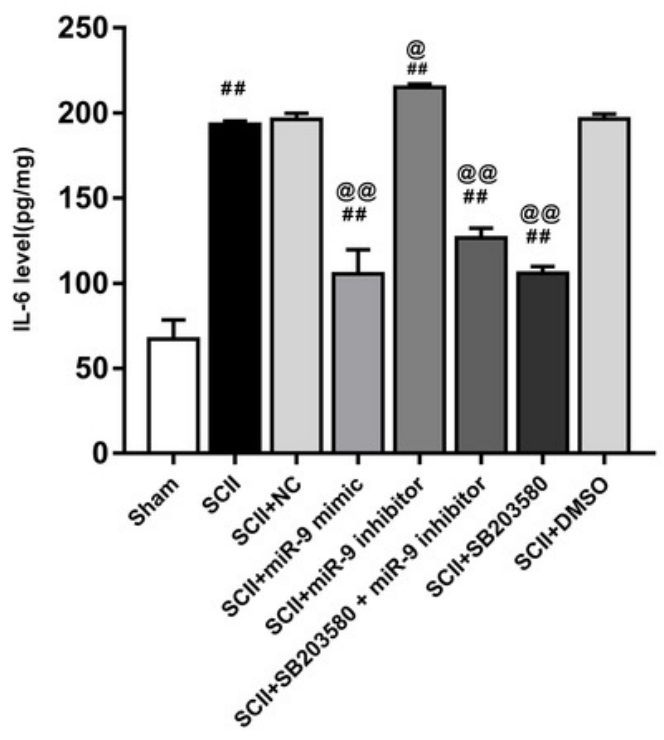


Figure 7

Figure 7. Intrathecal pretreatment with miR-9 mimic or Notch2 siRNA ameliorates neurological function and reduces the increase of cleaved-caspase3 after SCII.

(A) Neurological function scores at $48 \mathrm{~h}$ after SCll in eight groups. Each symbol represents one rat $(n=8)$. (B-D) Representative Western blotting and the protein expression of Notch2 and cleaved-caspase-3 at $48 \mathrm{~h}$ after SCII in eight groups $(\mathrm{n}=3) .{ }^{*} p<0.05,{ }^{\# \#} p<0.01$, versus group sham. ${ }^{\circledR} p<0.05,{ }^{@} p<0.01$, versus group SCII.

A

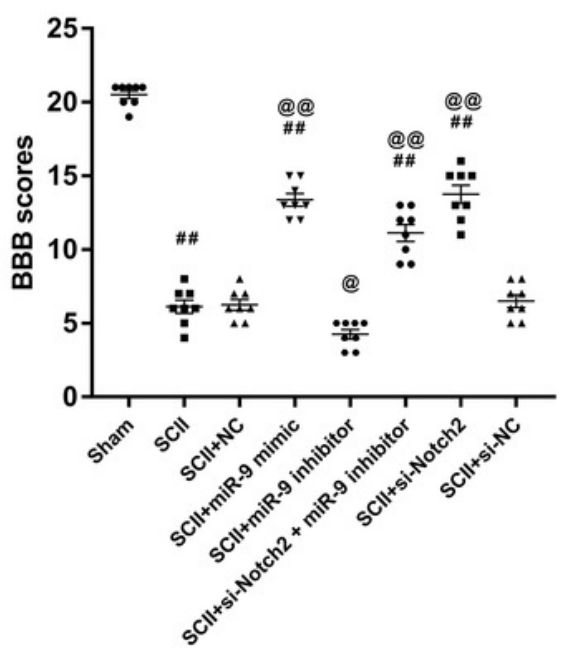

C

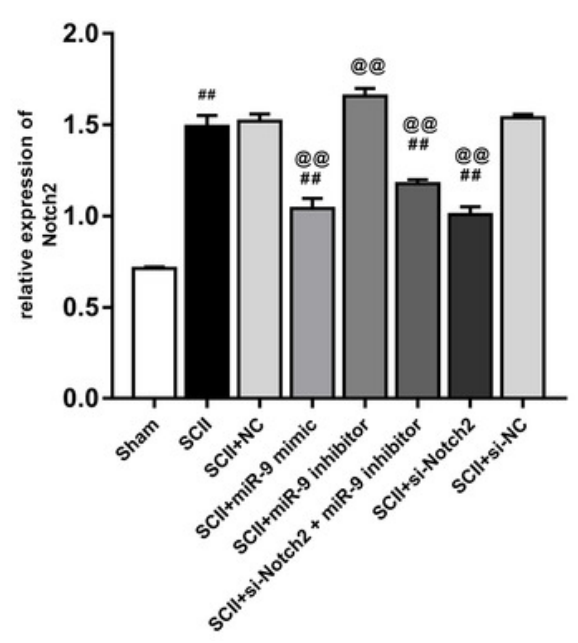

B

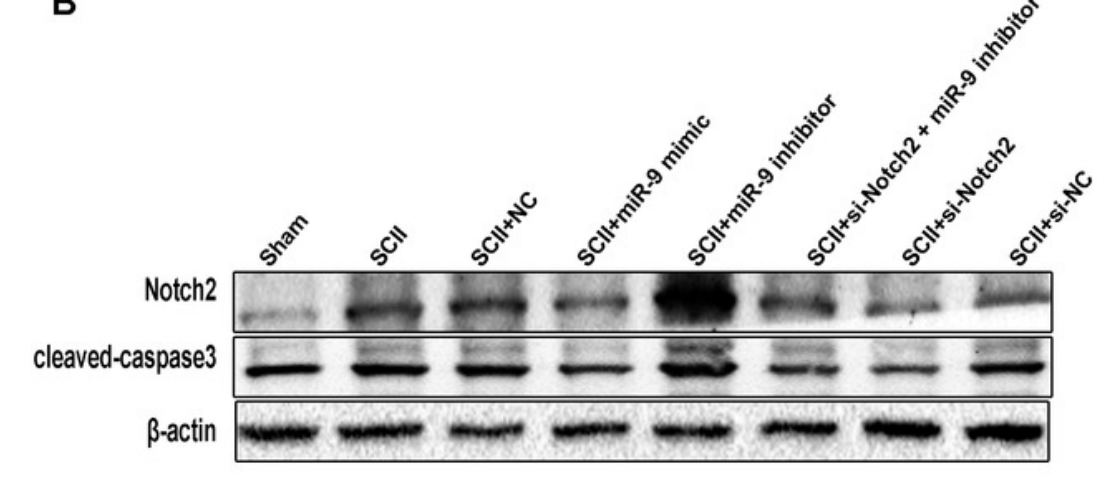

D

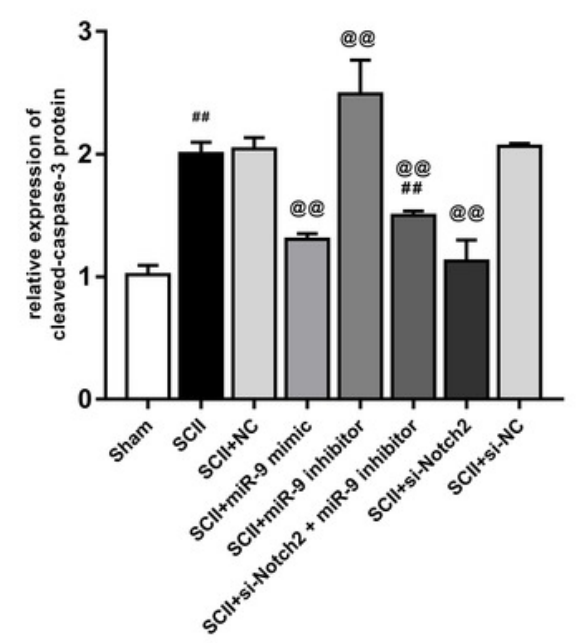




\section{Table 1 (on next page)}

Table 1. KEGG Pathway Terms for potential targets of miR-9 $(p<0.05)$.

* MAP2K3 and Notch2 were enriched in MAPK signaling pathway and Notch signaling pathway, respectively. 
3 Table 1. KEGG Pathway Terms for potential targets of miR-9 $(p<0.05)$

\begin{tabular}{|l|l|l|}
\hline KEGG PATHWAY Term & $\boldsymbol{P}$ Value & Genes \\
\hline MAPK signaling pathway & 0.004217403 & $\begin{array}{l}\text { MAP2K3*, TGFBR2, PDGFRB, NFKB1, MAPKAPK2, SRF, } \\
\text { STK3 }\end{array}$ \\
\hline Focal adhesion & 0.008281511 & DIAPH1, PDGFRB, SHC1, THBS2, SRC, VCL \\
\hline Prolactin signaling pathway & 0.010146632 & NFKB1, SHC1, SOCS5, SRC \\
\hline Notch signaling pathway & 0.033001048 & HES1, NOTCH2*, NCOR2 \\
\hline
\end{tabular}

$4 *$ MAP2K3 and Notch2 were enriched in MAPK signaling pathway and Notch signaling

5 pathway, respectively. 